\title{
Multidimensional Generalized Gini Indices
}

\author{
Thibault Gajdos \\ CNRS-CREST, \\ 15 boulevard Gabriel Peri, \\ 92245 Malakoff Cedex, France \\ (e-mail: gajdos@ensae.fr) \\ and \\ JOHN A. WEYMARK \\ Department of Economics, Box 1819, Station B, \\ Vanderbilt University, Nashville, TN 37235, U.S.A. \\ (e-mail: john.weymark@vanderbilt.edu)
}

May 2003

Revised, July 2003

\begin{abstract}
The axioms used to characterize the generalized Gini social evaluation orderings for one-dimensional distributions are extended to the multidimensional attributes case. A social evaluation ordering is shown to have a two-stage aggregation representation if these axioms and a separability assumption are satisfied. In the first stage, the distributions of each attribute are aggregated using generalized Gini social evaluation functions. The functional form of the second-stage aggregator depends on the number of attributes and on which version of a comonotonic additivity axiom is used. The implications of these results for the corresponding multidimensional indices of relative and absolute inequality are also considered.
\end{abstract}

Keywords and Phrases: Generalized Gini, multidimensional inequality.

JEL Classification Number: D63. 


\section{Introduction}

An individual's well-being depends on many factors, such as income, life expectancy, and health status. Because of the multidimensional nature of wellbeing, univariate indices of income inequality may give a misleading picture of the extent of inequality within a given population or between different groups of individuals. The formal analysis of multidimensional inequality has its origins in a pioneering article by Kolm (1977). ${ }^{1}$ In Kolm's article and in the later work of Atkinson and Bourguignon (1982), the focus is on developing dominance criteria that can be used to determine when one multidimensional distribution exhibits more inequality than another. Kolm provided a number of multi-attribute generalizations of the Pigou-Dalton transfer principle, whereas Atkinson and Bourguignon proposed dominance principles that take account of the positive dependence between the distributions of the different components of well-being.

The rankings generated by these dominance principles are incomplete. In many circumstances, it is useful to have an index of inequality that can be used to compare any pair of distributions. In the normative approach to inequality measurement, an inequality index is constructed from a social evaluation ordering (or its representation, a social evaluation function) of the possible distributions. ${ }^{2}$ For univariate distributions, the most commonlyused procedure for deriving a relative (i.e., scale invariant) inequality index from a social evaluation ordering was independently proposed by Atkinson (1970) and Kolm (1969) and popularized by Sen (1973). Kolm (1969) also proposed a procedure for deriving an absolute (i.e., translation invariant) inequality index from a social evaluation ordering. ${ }^{3}$ Multi-attribute generalizations of the univariate Atkinson-Kolm-Sen and Kolm methodologies have been proposed by Kolm (1977) and Tsui (1995), respectively.

The normative approach to inequality measurement has been used by Tsui (1995) to develop multi-attribute generalizations of the univariate Atkinson (1970) and Kolm (1969)-Pollak (1971) inequality indices. Tsui constructed his indices by axiomatically characterizing multi-attribute Atkinson and Kolm-Pollak classes of social evaluation functions. Tsui's axioms are

\footnotetext{
${ }^{1}$ For surveys of the literature on multidimensional inequality, see Maasoumi (1999), Savaglio (2002), and Weymark (2003).

${ }^{2} \mathrm{~A}$ social evaluation ordering is sometimes called a social welfare ordering.

${ }^{3}$ See Blackorby, Bossert, and Donaldson (1999) and Dutta (2002) for surveys of the normative approach to the measurement of univariate inequality.
} 
multivariate generalizations of the axioms used by Blackorby, Donaldson, and Auersperg (1981) to characterize the social evaluation functions underlying the univariate Atkinson and Kolm-Pollak inequality indices. ${ }^{4}$

The social evaluation functions identified by Tsui have the feature that they can be constructed in two steps. A utility function is first used to determine the utility of each person's allocation and then these utilities are aggregated (using simple summation) to provide the overall evaluation. Maasoumi (1986) had earlier suggested constructing a multi-attribute inequality index directly by first using a utility function to generate a distribution of utilities and then applying a univariate index of inequality to this distribution to obtain the multi-attribute index's value. However, as pointed out by Dardanoni (1995) (see also Weymark (2003)), by applying this two-step procedure to an inequality index, rather than to a social evaluation function, the resulting measure may fail to satisfy a multi-attribute version of the Pigou-Dalton transfer principle. ${ }^{5}$

In this article, we follow the general approach of Tsui (1995), but consider a different set of axioms. We extend the axioms used by Weymark (1981) to characterize the generalized Gini social evaluation orderings for one-dimensional distributions to the multi-attributes case. We show that a social evaluation ordering has a two-stage aggregation representation if these axioms and a separability assumption are satisfied. In the first stage, the distributions of each attribute are aggregated using univariate generalized Gini social evaluation functions. The functional form of the second-stage aggregator depends on the number of attributes and on which version of a comonotonic additivity axiom is used. Note that the order in which individuals and attributes are aggregated is the reverse of the order used by Tsui (1995). We also determine which of our orderings satisfy the invariance assumptions needed to generate relative and absolute indices of inequality. The implications of these results for the functional forms of the corresponding multidimensional indices of relative and absolute inequality are also considered. Finally, we show that our separability axioms are inconsistent with a correlation increasing majorization axiom proposed by Tsui (1999) when individuals are treated symmetrically.

\footnotetext{
${ }^{4}$ Tsui (1999) has also axiomatized a class of multi-attribute generalized entropy relative inequality indices. However, these inequality indices are characterized directly, rather than indirectly using social evaluation functions.

${ }^{5}$ List (1999) has proposed an alternative multi-stage procedure for constructing multiattribute inequality indices that avoids the problem identified by Dardanoni.
} 


\section{Preliminaries}

The set of individuals is $N=\{1, \ldots, n\}$, with $n \geq 2$. There are $q$ attributes of well-being, with $q \geq 2$. The set of attributes is $Q=\{1, \ldots, q\}$. In addition to income, examples of possible attributes are measures of educational attainment, health status, and longevity. The $q$ attributes could be incomes in different states of the world, in which case we are concerned with inequality under uncertainty, as in Ben-Porath, Gilboa, and Schmeidler (1997) and Gajdos and Maurin (2002). Alternatively, the attributes could be incomes in different time periods.

An allocation of attributes among the population is an $n \times q$ real-valued matrix. A generic element of an allocation matrix $X$ is $x_{i j}$, the quantity of attribute $j$ allocated to individual $i$. The $i^{\text {th }}$ row of a matrix $X$ is denoted $x_{i}$, whereas its $j^{\text {th }}$ column is denoted $x_{. j}$. For all $Q_{0} \subseteq Q$ for which $Q_{0} \neq \varnothing$, let $X_{Q_{0}}$ be the sub-allocation matrix of the attributes in $Q_{0}$. For any $j \in Q$, if $Q_{0}=\{j\}$, we write $x_{\cdot j}$ instead of $X_{\{j\}}$, and if $Q_{0}=\{1, \ldots, j-1, j+1, \ldots, q\}$, we write $X_{-j}$ instead of $X_{Q_{0}}$. Let $\mathcal{Q}$ be the set of ordered bi-partitions of $Q$. Formally,

$$
\mathcal{Q}=\left\{\left(Q_{1}, Q_{2}\right) \subseteq Q \times Q \mid Q_{1} \cup Q_{2}=Q, Q_{1} \cap Q_{2}=\varnothing, Q_{1} \neq \varnothing, Q_{2} \neq \varnothing\right\} .
$$

For all $\left(Q_{1}, Q_{2}\right) \in \mathcal{Q}$, it is sometimes convenient to let $\left(X_{Q_{1}}, X_{Q_{2}}\right)$ denote the matrix $X$.

The set of all allocation matrices is $\mathcal{M}$ and the set of all allocation matrices whose elements are nonnegative is $\mathcal{M}_{+}$. Let $\mathcal{M}_{+}^{*}$ denote the set of allocation matrices in $\mathcal{M}_{+}$that have at least one positive element in each column. These three classes of allocation matrices are the multidimensional analogues of the standard domains used for univariate distributions of incomes. We also need to consider nonnegative allocation matrices that only differ from the null matrix in one column. Let $\mathcal{M}_{+}^{* *}$ denote this class of allocation matrices. The allocation matrix whose elements are all equal to 0 (resp. 1) is $\mathbf{0}$ (resp. 1).

A social evaluation is a binary relation $\succeq$ on a set of allocation matrices $\mathcal{D}$. The relation $\succeq$ is interpreted as meaning "weakly socially preferred to". The symmetric and asymmetric factors of $\succeq$ are $\sim$ and $\succ$, respectively. We assume that $\mathcal{D} \in\left\{\mathcal{M}, \mathcal{M}_{+}, \mathcal{M}_{+}^{*}\right\}$. If not stated explicitly, $\mathcal{D}$ can be any one of these three sets. An allocation matrix $X$ is nonincreasing comonotonic if $x_{1 j} \geq x_{2 j} \geq \cdots \geq x_{n j}$ for all $j \in Q$. Let $\mathcal{D}^{\mathrm{D}}$ denote the set of nonincreasing comonotonic matrices in $\mathcal{D}$. 
For any $x \in \mathbb{R}^{n}, \tilde{x}$ is the permutation of $x$ for which $\tilde{x}_{1} \geq \tilde{x}_{2} \geq \cdots \geq \tilde{x}_{n}{ }^{6}$ Let $\mathbb{R}_{+}^{n *}=\mathbb{R}_{+}^{n} \backslash\{(0, \ldots, 0)\}$. For $\mathbb{D} \in\left\{\mathbb{R}^{n}, \mathbb{R}_{+}^{n}, \mathbb{R}_{+}^{n *}\right\}$, a generalized Gini social evaluation function is a function $g: \mathbb{D} \rightarrow \mathbb{R}$ for which

$$
g(x)=\sum_{i=1}^{n} a_{i} \tilde{x}_{i}, \forall x \in \mathbb{D}
$$

where $0<a_{1} \leq a_{2} \leq \cdots \leq a_{n}$ and $\sum_{i=1}^{n} a_{i}=1$. The Gini social evaluation function is the special case of (1) in which $a_{i}=(2 i-1) / n^{2}$ for all $i \in N$.

For any $x \in \mathbb{R}^{n}, \mu(x)$ is the mean of $x$. For any $X \in \mathcal{D}$, we let $X_{\mu}$ denote the matrix for which every entry in the $j^{\text {th }}$ column is equal to $\mu\left(x_{\cdot j}\right)$.

\section{Multidimensional Generalized Gini Axioms}

In this section, we introduce the axioms that are used in Section 4 to characterize a number of classes of multidimensional generalized Gini social evaluation orderings. The first axiom requires $\succeq$ to be a complete preorder.

Ordering (ORD). The binary relation $\succeq$ is reflexive, complete, and transitive on $\mathcal{D}$.

The next axiom says that a strict ranking of two allocation matrices $X$ and $Y$ is invariant to small perturbations of these matrices.

Continuity (CONT). The sets $\{Y \in \mathcal{D} \mid Y \succ X\}$ and $\{Y \in \mathcal{D} \mid X \succ Y\}$ are open for all $X \in \mathcal{D}$. $^{7}$

The monotonicity axiom says that if $X$ is obtained from $Y$ by increasing at least one person's allocation of some attribute without decreasing anyone's allocation of any attribute, then $X$ is strictly preferred to $Y$.

Monotonicity (MON). For all $X, Y \in \mathcal{D}$,

$$
\left[\left(x_{i j} \geq y_{i j}, \forall(i, j) \in N \times Q\right) \&(X \neq Y)\right] \Rightarrow X \succ Y \text {. }
$$

\footnotetext{
${ }^{6} \mathbb{R}, \mathbb{R}_{+}$, and $\mathbb{R}_{++}$denote the set of real numbers, nonnegative real numbers, and positive real numbers, respectively.

${ }^{7} \mathrm{~A}$ matrix in $\mathcal{D}$ can be thought of as a vector in $\mathbb{R}^{n q}$. A subset of $\mathcal{D}$ is open if the corresponding set of vectors is open in $\mathbb{R}^{n q}$.
} 
The anonymity axiom requires $\succeq$ to treat individuals symmetrically. It says that it is a matter of social indifference if the individual allocations are permuted.

Anonymity (ANON). For all $n \times n$ permutation matrices $\Pi$ and all $X \in \mathcal{D}$, $X \sim \Pi X$.

In order for a social evaluation ordering on univariate distributions of income to serve as a satisfactory foundation for an inequality index, it should satisfy the Pigou (1912)-Dalton (1920) transfer principle. A Pigou-Dalton transfer is a transfer of income from a richer to a poorer individual that diminishes the absolute value of the difference between their incomes. The (weak form of the) Pigou-Dalton transfer principle says that if distribution $x$ can be obtained from distribution $y$ by a sequence of Pigou-Dalton transfers, then $x$ is socially weakly preferred to $y$.

A number of different multi-attribute generalizations of the Pigou-Dalton transfer principle have been proposed. See Kolm (1977), Marshall and Olkin (1979), and Savaglio (2002). We consider two of them.

After a Pigou-Dalton transfer of income between two individuals, the income of each of these individuals is a convex combination of the two pretransfer incomes. In the multi-attribute case, a univariate Pigou-Dalton transfer can be applied to each attribute. When, for a given pair of individuals, the same convex combinations are used for each attribute, we have a uniform Pigou-Dalton transfer.

Definition. For all $X, Y \in \mathcal{D}, X$ is obtained from $Y$ by a uniform PigouDalton transfer if $X \neq Y$ and there exist $i_{1}$ and $i_{2}$ in $N$ and $\lambda \in(0,1)$ such that (i) $x_{i_{1} j}=\lambda y_{i_{1} j}+(1-\lambda) y_{i_{2} j}$ for all $j \in Q$, (ii) $x_{i_{2} j}=(1-\lambda) y_{i_{1} j}+\lambda y_{i_{2} j}$ for all $j \in Q$, and (iii) $x_{i}$. $=y_{i}$. for all $i \notin\left\{i_{1}, i_{2}\right\}$.

If allocation $X$ can be obtained from allocation $Y$ by a finite sequence of such transfers, then $X$ is said to uniformly Pigou-Dalton majorize $Y$.

Definition. For all $X, Y \in \mathcal{D}, X$ uniformly Pigou-Dalton majorizes $Y$, denoted $X \succ_{\mathrm{U}} Y$, whenever $X$ can be obtained from $Y$ by a finite sequence of uniform Pigou-Dalton transfers.

The binary relation $\succ_{\mathrm{U}}$ is a partial order of the allocation matrices in $\mathcal{D}$. This partial order is used to define our first multi-attribute Pigou-Dalton transfer principle. 
Weak Uniform Pigou-Dalton Majorization (WUPM). For all $X, Y \in$ $\mathcal{D}$,

$$
X \succ_{\mathrm{U}} Y \Rightarrow X \succeq Y
$$

In the univariate case, distribution $x$ can be obtained from distribution $y$ by a finite sequence of Pigou-Dalton transfers if and only if $x$ can be obtained by premultiplying $y$ by a bistochastic matrix that is not a permutation matrix. ${ }^{8}$ Uniform majorization is the multi-attribute analogue of this construction.

Definition. For all $X, Y \in \mathcal{D}, X$ uniformly majorizes $Y$, denoted $X \succ_{\mathrm{B}} Y$, if there exists an $n \times n$ bistochastic matrix $B$ such that $X=B Y$ and $X$ is not a permutation of the rows of $Y$.

The partial order $\succ_{\mathrm{B}}$ is used to define our second multi-attribute PigouDalton transfer principle.

Weak Uniform Majorization (WUM). For all $X, Y \in \mathcal{D}$,

$$
X \succ_{\mathrm{B}} Y \Rightarrow X \succeq Y \text {. }
$$

As noted above, the univariate analogues of the partial orders $\succ_{\mathrm{U}}$ and $\succ_{\mathrm{B}}$ are equivalent. This equivalence also holds if $n=2$ when $q \geq 2$. However, if $q \geq 2$ and $n \geq 3, X \succ_{\mathrm{U}} Y$ implies $X \succ_{\mathrm{B}} Y$, but the reverse implication need not hold. As a consequence, Weak Uniform Majorization is a stronger condition than Weak Uniform Pigou-Dalton Majorization. See Kolm (1977) and Marshall and Olkin (1979). ${ }^{9}$

Weymark (1981) introduced a comonotonic additivity axiom for the ranking of one-dimensional income distributions. It requires the ranking of two comonotonic distributions to be invariant to the addition of a common distribution to both of these distributions provided that the third distribution is also comonotonic with the original distributions. The rationale offered for this axiom is that each person's income may come from a number of different

\footnotetext{
${ }^{8}$ See, for example, Hardy, Littlewood, and Pólya (1934) or Marshall and Olkin (1979). A nonnegative square matrix is bistochastic if all of its row and column sums are equal to 1.

${ }^{9}$ The terminology used here is based on Tsui (1999). In the terminology of Marshall and Olkin (1979), $X \succ_{\mathrm{U}} Y$ is equivalent to saying that $Y$ chain majorizes $X$ and $X \succ_{\mathrm{B}} Y$ is equivalent to saying that $Y$ majorizes $X$.
} 
sources (wages, interest, etc.) and if the incomes from all but once source of income are the same in the two distributions of total income, then the ranking of these distributions should only depend on the distributions of income from the variable source.

We consider two multi-attribute extensions of this axiom. In both cases, we require the ranking of two nonincreasing comonotonic allocation matrices $X$ and $Y$ by $\succeq$ to be invariant to the common addition of a third allocation matrix $Z$ that is nonincreasing comonotonic with respect to both $X$ and $Y$. Our Weak Comonotonic Additivity axiom applies if $X$ and $Y$ differ in only one attribute and $Z$ only has non-zero values for this attribute. In our Strong Comonotonic Additivity axiom, the distributions of any attribute in the three allocation matrices $X, Y$, and $Z$ are permitted to be different from one another.

Weak Comonotonic Additivity (WCA). For all $X, Y \in \mathcal{D}^{\mathrm{D}}$ and all $Z \in \mathcal{D}^{\mathrm{D}} \cup \mathcal{M}_{+}^{* * \mathrm{D}}$ for which there exists a $j_{0} \in N$ such that (i) $x_{\cdot j}=y_{\cdot j}$ for all $j \neq j_{0}$ and (ii) $z_{i j}=0$ for all $i \in N$ and all $j \neq j_{0}$,

$$
X \succeq Y \Leftrightarrow X+Z \succeq Y+Z \text {. }
$$

Strong Comonotonic Additivity (SCA). For all $X, Y \in \mathcal{D}^{\mathrm{D}}$ and all $Z \in \mathcal{D}^{\mathrm{D}} \cup \mathcal{M}_{+}^{* * \mathrm{D}}$,

$$
X \succeq Y \Leftrightarrow X+Z \succeq Y+Z
$$

Note that if the domain is $\mathcal{M}_{+}^{*}$, the allocation matrix $Z$ that is added to both $X$ and $Y$ is not in the domain if $Z \in \mathcal{M}_{+}^{* * D}$. However, it is nevertheless the case that $X+Z$ and $Y+Z$ are in the domain, which is all that is needed for our comonotonic additivity principles to apply.

All of the preceding axioms are multi-attribute extensions of axioms for one-dimensional distributions. Their one-dimensional counterparts characterize the class of generalized Gini social evaluation functions with positive weights. See Weymark (1981). ${ }^{10}$

If the conditional ordering of some subset of the variables obtained by fixing the values of the remaining variables is independent of the values of

\footnotetext{
${ }^{10}$ Weymark (1981) axiomatized the class of generalized Gini absolute inequality indices. It is straightforward to modify his analysis in order to obtain an axiomatization of the class of generalized Gini social evaluation functions. He did not employ a monotonicity assumption. As a consequence, the weights in his representation theorem need not be positive.
} 
the conditioning variables, then the first set of variables is said to be separable from the second. The next two axioms are concerned with the separability of $\succeq$ across attributes. In the subsequent discussion, when we say that the set of attributes $Q_{1} \subseteq Q$ is separable from the complementary set of attributes, we mean that the set of all variables $i j$ for which $i \in N$ and $j \in Q_{1}$ is separable from the variables associated with the attributes not in $Q_{1}$. Weak Attribute Separability requires that there exist some attribute that is separable from the other attributes. Strong Attribute Separability strengthens this condition by requiring any subset of the attributes to be separable from the other attributes. $^{11}$

Weak Attribute Separability (WSEP). There exists $j_{0} \in Q$ such that for all $x_{\cdot j_{0}}, y_{\cdot j_{0}}, \bar{X}_{-j_{0}}$, and $\bar{Z}_{-j_{0}}$,

$$
\left(x_{\cdot j_{0}}, \bar{X}_{-j_{0}}\right) \succeq\left(y_{\cdot j_{0}}, \bar{X}_{-j_{0}}\right) \Leftrightarrow\left(x_{\cdot j_{0}}, \bar{Z}_{-j_{0}}\right) \succeq\left(y_{\cdot j_{0}}, \bar{Z}_{-j_{0}}\right) .
$$

Strong Attribute Separability (SSEP). For all $\left(Q_{1}, Q_{2}\right) \in \mathcal{Q}$ and all $X_{Q_{1}}, Y_{Q_{1}}, \bar{X}_{Q_{2}}$, and $\bar{Z}_{Q_{2}}$,

$$
\left(X_{Q_{1}}, \bar{X}_{Q_{2}}\right) \succeq\left(Y_{Q_{1}}, \bar{X}_{Q_{2}}\right) \Leftrightarrow\left(X_{Q_{1}}, \bar{Z}_{Q_{2}}\right) \succeq\left(Y_{Q_{1}}, \bar{Z}_{Q_{2}}\right) .
$$

\section{Multidimensional Generalized Gini Social Evaluation Orderings}

In this section, we show that if multi-attribute versions of the axioms that characterize the class of generalized Gini social evaluation orderings for univarate distributions are combined with SSEP, then the social evaluation ordering can be represented by a two-stage aggregator function. In the first stage, the distributions of each attribute are aggregated using a univariate generalized Gini social evaluation function. The weights in these functions can be attribute specific. In the second stage, the values of these generalized Ginis are aggregated. The functional form of the second-stage aggregator depends on which version of our comonotonic additivity axiom is used and on the number of attributes.

\footnotetext{
${ }^{11}$ In order to avoid introducing even more notation, we do not explicitly state the domains for the various variables that appear in the statement of the two separability axioms. Each of the allocation matrices considered in these axioms must be in $\mathcal{D}$. See Blackorby, Primont, and Russell (1978) for further discussion of these separability axioms.
} 
Our characterization theorems do not depend on whether our multiattribute generalization of the Pigou-Dalton transfer principle is WUPM or WUM. In general, WUPM and WUM are not equivalent if $q \geq 2$. However, in the presence of ANON and SSEP, the implications of WUPM and WUM can be determined one attribute at a time. Consequently, as we show in Theorem 1, WUPM and WUM place equivalent restrictions on a social evaluation ordering if it satisfies ANON and SSEP.

Theorem 1. Suppose that $\mathcal{D} \in\left\{\mathcal{M}, \mathcal{M}_{+}, \mathcal{M}_{+}^{*}\right\}$. If the binary relation $\succeq$ on $\mathcal{D}$ satisfies $\mathrm{SSEP}$ and $\mathrm{ANON}$, then $\succeq$ satisfies WUM if and only if it satisfies WUPM.

Proof. Because $X \succ_{\mathrm{U}} Y$ implies $X \succ_{\mathrm{B}} Y$ for all $X, Y \in \mathcal{D}$, if $\succeq$ satisfies WUM, it also satisfies WUPM. Hence, we only need to show that the converse implication holds.

Suppose that $\succeq$ satisfies SSEP, ANON, and WUPM. Let $X, Y \in \mathcal{D}$ be such that $X \succ_{\mathrm{B}} Y$.

Let $\hat{X}^{1}=\left(x_{\cdot 1}, \mathbf{1}_{-1}\right)$ and $\hat{Y}^{1}=\left(y_{\cdot 1}, \mathbf{1}_{-1}\right)$. Two cases may arise. In the first case, $x_{\cdot 1}$ is a permutation of $y_{\cdot 1} \cdot{ }^{12}$ By ANON, it then follows that $\hat{X}^{1} \sim \hat{Y}^{1}$. In the second case, $x_{\cdot 1}$ is not a permutation of $y_{\cdot 1}$. Then, $\hat{X}^{1}$ is obtained from $\hat{Y}^{1}$ by a finite sequence of uniform Pigou-Dalton transfers. Therefore, because $\succeq$ satisfies WUPM, we have $\hat{X}^{1} \succeq \hat{Y}^{1}$. Hence, in both cases, $\hat{X}^{1} \succeq$ $\hat{Y}^{1}$. But this implies, by SSEP, that $X^{1} \succeq Y$, where $X^{1}=\left(x_{\cdot 1}, Y_{-1}\right)$.

By the same reasoning as above, we have $\left(x_{\cdot 2}, \mathbf{1}_{-2}\right) \succeq\left(y_{\cdot 2}, \mathbf{1}_{-2}\right)$, and therefore, by SSEP, that $X^{2} \succeq X^{1}$, where $X^{2}=\left(x_{\cdot 2}, X_{-2}^{1}\right)$. Because $X^{1} \succeq Y$, transitivity of $\succeq$ then implies that $X^{2} \succeq Y$. Note that $X^{2}=\left(X_{\{1,2\}}, Y_{Q \backslash\{1,2\}}\right)$.

By iterating this process on $Q$, we conclude that $X \succeq Y$.

In view of Theorem 1, it doesn't matter which of WUPM and WUM we include in our set of axioms. For concreteness, we use WUPM. In all of the characterization theorems in this section, we suppose that the social evaluation ordering $\succeq$ satisfies ORD, CONT, MON, ANON, WUPM, and SSEP.

As is well-known, the functional structure implications of separability axioms that operate on all ordered bi-partitions of a set of variables depend on whether the number of variables being partitioned is two or whether it is three or more. See, for example, Blackorby, Primont, and Russell (1978).

\footnotetext{
${ }^{12}$ Note that this case applies if $x_{\cdot 1}=y \cdot 1$.
} 
With SSEP, the variables being partitioned are the attributes. We first consider the case in which there are three or more attributes. When this is the case, Theorem 2 shows that $\succeq$ can be represented by a two-stage aggregator function, as described above, where the second-stage aggregator is a continuous, increasing additive function of the generalized Gini aggregators used in the first stage if WCA is added to the six axioms listed in the preceding paragraph.

Theorem 2. Suppose that $\mathcal{D}=\mathcal{M}$ (resp. $\mathcal{D}=\mathcal{M}_{+}$, resp. $\left.\mathcal{D}=\mathcal{M}_{+}^{*}\right)$. If $q>2$, then the binary relation $\succeq$ on $\mathcal{D}$ satisfies ORD, CONT, MON, ANON, WUPM, SSEP, and WCA if and only if there exists an $n \times q$ matrix $A$ of positive coefficients with $a_{. j}$ nondecreasing and $\sum_{i=1}^{n} a_{i j}=1$ for all $j \in Q$ and there exist $q$ continuous increasing functions $v_{j}: \mathbb{R} \rightarrow \mathbb{R}$ (resp. $v_{j}: \mathbb{R}_{+} \rightarrow \mathbb{R}$, resp. $\left.v_{j}: \mathbb{R}_{++} \rightarrow \mathbb{R}\right)$ such that

$$
X \succeq Y \Leftrightarrow \sum_{j=1}^{q} v_{j}\left(\sum_{i=1}^{n} a_{i j} \tilde{x}_{i j}\right) \geq \sum_{j=1}^{q} v_{j}\left(\sum_{i=1}^{n} a_{i j} \tilde{y}_{i j}\right), \forall X, Y \in \mathcal{D} .
$$

Furthermore, the functions $v_{j}$ are unique up to a common increasing affine transformation and the matrix of coefficients $A$ is unique.

Proof. The necessity part of the theorem is straightforward to verify. We therefore only prove sufficiency. We establish the theorem for the domain $\mathcal{D}=\mathcal{M}{ }^{13}$

By Theorem I in Debreu (1954), we know that if $\succeq$ satisfies ORD, CONT, and MON, then there exists a continuous, increasing function $f: \mathcal{D} \rightarrow \mathbb{R}$ that represents $\succeq$. Furthermore, for all $j \in Q$, SSEP implies that the $j^{\text {th }}$ attribute is separable from the the complementary set of attributes. Note that MON implies that every attribute is essential. ${ }^{14}$ Because $q>2$, Theorem 3 in Debreu (1960) applies. Hence, there exist $q$ continuous and increasing functions $U_{j}: \mathbb{R}^{n} \rightarrow \mathbb{R}$ such that

$$
X \succeq Y \Leftrightarrow \sum_{j=1}^{q} U_{j}\left(x_{\cdot j}\right) \geq \sum_{j=1}^{q} U_{j}\left(y_{\cdot j}\right), \forall X, Y \in \mathcal{D} .
$$

\footnotetext{
${ }^{13}$ For the domain $\mathcal{M}_{+}\left(\right.$resp. $\left.\mathcal{M}_{+}^{*}\right), \mathbb{R}^{n}$ must be changed to $\mathbb{R}_{+}^{n}$ (resp. $\mathbb{R}_{+}^{n *}$ ) throughout the proof and the function $v_{j}$ is defined on $\mathbb{R}_{+}$(resp. $\mathbb{R}_{++}$), but otherwise the argument is identical.

${ }^{14}$ The $j^{\text {th }}$ attribute is essential if there exist values for the allocations of the other attributes such that the conditional ordering of the allocations of the $j^{\text {th }}$ attribute is not the trivial one in which all allocations are indifferent to each other.
} 
Furthermore, the functions $U_{j}$ are unique up to a common increasing affine transformation.

Consider any $X \in \mathcal{D}$. For all $j \in Q$, we define the binary relation $\succeq_{j}$ on $\mathbb{R}^{n}$ by setting

$$
y_{\cdot j} \succeq_{j} z_{\cdot j} \Leftrightarrow\left(X_{-j}, y_{\cdot j}\right) \succeq\left(X_{-j}, z_{\cdot j}\right), \forall y_{\cdot j}, z_{\cdot j} \in \mathbb{R}^{n} .
$$

Because of SSEP, $\succeq_{j}$ does not depend on the choice of the matrix $X$. For all $j \in Q$, (3) implies that $\succeq_{j}$ can be represented by $U_{j}$.

Consider any $j \in Q$. Because $\succeq$ satisfies CONT and ANON, $\succeq_{j}$ is a continuous, symmetric ordering of $\mathbb{R}^{n}$. Now, consider any $y_{\cdot j}, z_{\cdot j}, t_{\cdot j} \in \mathbb{R}^{n}$ and any $X \in \mathcal{D}^{\mathrm{D}}$. By $(4), \tilde{y}_{\cdot j} \succeq_{j} \tilde{z}_{\cdot j}$ implies $\left(X_{-j}, \tilde{y}_{\cdot j}\right) \succeq\left(X_{-j}, \tilde{z}_{\cdot j}\right)$. Note that $\left(X_{-j}, \tilde{y}_{\cdot j}\right)$ and $\left(X_{-j}, \tilde{z}_{\cdot j}\right)$ both belong to $\mathcal{D}^{\mathrm{D}}$. Applying WCA, we obtain

$$
\left(X_{-j}, \tilde{y}_{\cdot j}\right) \succeq\left(X_{-j}, \tilde{z}_{\cdot j}\right) \Leftrightarrow\left(X_{-j}, \tilde{y}_{\cdot j}\right)+\left(\mathbf{0}_{-j}, \tilde{t}_{\cdot j}\right) \succeq\left(X_{-j}, \tilde{z}_{\cdot j}\right)+\left(\mathbf{0}_{-j}, \tilde{t}_{\cdot j}\right) .
$$

Equivalently,

$$
\left(X_{-j}, \tilde{y}_{\cdot j}\right) \succeq\left(X_{-j}, \tilde{z}_{\cdot j}\right) \Leftrightarrow\left(X_{-j}, \tilde{y}_{\cdot j}+\tilde{t}_{\cdot j}\right) \succeq\left(X_{-j}, \tilde{z}_{\cdot j}+\tilde{t}_{\cdot j}\right) .
$$

We have thus shown that

$$
\tilde{y}_{\cdot j} \succeq_{j} \tilde{z}_{\cdot j} \Leftrightarrow \tilde{y}_{\cdot j}+\tilde{t}_{\cdot j} \succeq_{j} \tilde{z}_{\cdot j}+\tilde{t}_{\cdot j}, \forall y_{\cdot j}, z_{\cdot j}, t_{\cdot j} \in \mathbb{R}^{n} .
$$

Therefore, $\succeq_{j}$ satisfies Axiom 4 in Weymark (1981). It then follows from MON and Theorem 3 in Weymark (1981) that there exist $a_{i j}>0, i \in N$, such that

$$
\tilde{x}_{\cdot j} \succeq_{j} \tilde{y}_{\cdot j} \Leftrightarrow \sum_{i=1}^{n} a_{i j} \tilde{x}_{i j} \geq \sum_{i=1}^{n} a_{i j} \tilde{y}_{i j}, \forall x_{\cdot j}, y_{\cdot j} \in \mathbb{R}^{n} \cdot 15
$$

We adopt the normalization $\sum_{i} a_{i j}=1$, which implies that the sequence of weights $\left(a_{i j}\right)_{i}$ is unique. WUPM implies that $\succeq_{j}$ satisfies the unidimensional Pigou-Dalton transfer principle, which in turn implies that $a_{1 j} \leq a_{2 j} \leq \cdots \leq$ $a_{n j}$.

Define the function $G_{j}: \mathbb{R}^{n} \rightarrow \mathbb{R}$ by setting

$$
G_{j}\left(x_{\cdot j}\right)=\sum_{i=1}^{n} a_{i j} \tilde{x}_{i j}, \forall x_{\cdot j} \in \mathbb{R}^{n} .
$$

\footnotetext{
$\mathbb{R}^{n}$.

${ }^{15}$ Weymark's theorem is for vectors in $\mathbb{R}_{+}^{n}$, but, as he notes, it also holds for vectors in
} 
Because $\succeq_{j}$ is symmetric, (5) implies that $G_{j}$ is a continuous representation of $\succeq_{j}$. Because $U_{j}$ is also a continuous representation of $\succeq_{j}$, there exists a continuous, increasing function $v_{j}: \mathbb{R} \rightarrow \mathbb{R}$ such that $U_{j}=v_{j} \circ G_{j}$.

The preceding argument holds for all $j \in Q$. It then follows that (3) can be rewritten as (2). Because the functions $U_{j}$ are unique up to a common increasing affine transformation, so are the functions $v_{j}$.

SSEP and ORD imply that the conditional ordering of the distributions of any attribute are independent of the values of the other variables. The proof of Theorem 2 shows that the properties of these orderings that are inherited from the axioms of the theorem are exactly those properties that are used to characterize the univariate generalized Ginis. This accounts for the functional structure of the first-stage aggregators. The additive structure of the second-stage aggregator follows from a standard separability theorem for three or more variables.

In Theorem 3, we consider the same set of axioms as in Theorem 2, but now suppose that there are only two attributes. Reasoning as in the proof of Theorem 2, the first-stage aggregators are generalized Ginis. However, because the separability axiom only operates on bi-partitions of two attributes, we can no longer conclude that the second-stage aggregator is additive.

Theorem 3. Suppose that $\mathcal{D}=\mathcal{M}$ (resp. $\mathcal{D}=\mathcal{M}_{+}$, resp. $\left.\mathcal{D}=\mathcal{M}_{+}^{*}\right)$. If $q=2$, then the binary relation $\succeq$ on $\mathcal{D}$ satisfies ORD, CONT, MON, ANON, WUPM, SSEP, and WCA if and only if there exists an $n \times 2$ matrix $A$ of positive coefficients with $a_{\cdot j}$ nondecreasing and $\sum_{i=1}^{n} a_{i j}=1$ for all $j \in\{1,2\}$ and there exists a continuous increasing function $V: \mathbb{R}^{2} \rightarrow \mathbb{R}$ (resp. $V: \mathbb{R}_{+}^{2} \rightarrow \mathbb{R}$, resp $V: \mathbb{R}_{++}^{2} \rightarrow \mathbb{R}$ ) such that

$$
\begin{aligned}
& X \succeq Y \Leftrightarrow V\left(\sum_{i=1}^{n} a_{i 1} \tilde{x}_{i 1}, \sum_{i=1}^{n} a_{i 2} \tilde{x}_{i 2}\right) \geq V\left(\sum_{i=1}^{n} a_{i 1} \tilde{y}_{i 1}, \sum_{i=1}^{n} a_{i 2} \tilde{y}_{i 2}\right), \\
& \forall X, Y \in \mathcal{D} .
\end{aligned}
$$

Furthermore, the function $V$ is unique up to an increasing transformation and the matrix of coefficients $A$ is unique.

Proof. The necessity part of the theorem is straightforward to verify. We therefore only prove sufficiency. As in the proof of Theorem 2, we only consider the domain $\mathcal{M}$ as it is trivial to modify the proof so that it applies to the other two domains. 
We know from the proof of Theorem 2 that ORD, CONT, MON, and SSEP imply that $\succeq$ can be represented by a continuous, increasing function $f: \mathcal{D} \rightarrow \mathbb{R}$. Furthermore, for all $j \in Q$, the $j^{\text {th }}$ attribute is essential and separable from the the complementary set of attributes. Hence, by Lemma 1 in Gorman (1968), there exist three continuous increasing functions $U_{0}: U_{1}\left(\mathbb{R}^{2}\right) \times U_{2}\left(\mathbb{R}^{2}\right) \rightarrow \mathbb{R}, U_{1}: \mathbb{R}^{2} \rightarrow \mathbb{R}$, and $U_{2}: \mathbb{R}^{2} \rightarrow \mathbb{R}$ such that

$$
X \succeq Y \Leftrightarrow U_{0}\left(U_{1}\left(x_{\cdot 1}\right), U_{2}\left(x_{\cdot 2}\right)\right) \geq U_{0}\left(U_{1}\left(y_{\cdot 1}\right), U_{2}\left(y_{\cdot 2}\right)\right), \forall X, Y \in \mathcal{D} .
$$

For $j=1,2$, defining $\succeq_{j}$ as in (3), the same argument as the one used in the proof of Theorem 2 implies that $\succeq_{j}$ can be represented by $U_{j}$ and that there exist two nondecreasing series of positive weights $\left(a_{i j}\right)_{i}$ for which $\sum_{i=1}^{n} a_{i j}=1$ such that

$$
x_{\cdot j} \succeq_{j} y_{\cdot j} \Leftrightarrow G_{j}\left(x_{\cdot j}\right) \geq G_{j}\left(y_{\cdot j}\right), \forall x_{\cdot j}, y_{\cdot j} \in \mathbb{R}^{n},
$$

where $G_{j}$ is defined in (6). Furthermore, each of these series of weights is unique. Therefore, for $j=1,2$, there exists a nondecreasing continuous function $v_{j}: \mathbb{R} \rightarrow \mathbb{R}$ such that $U_{j}=v_{j} \circ G_{j}$. Substituting in (6), we obtain

$$
\begin{array}{r}
X \succeq Y \Leftrightarrow U_{0}\left(v_{1}\left(G_{j}\left(x_{\cdot 1}\right)\right), v_{2}\left(G_{j}\left(x_{\cdot 2}\right)\right) \geq U_{0}\left(v_{1}\left(G_{j}\left(y_{\cdot 1}\right)\right), v_{2}\left(G_{j}(y \cdot 2)\right)\right),\right. \\
\forall X, Y \in \mathcal{D} .
\end{array}
$$

Defining the function $V: \mathbb{R}^{2} \rightarrow \mathbb{R}$ by setting $V(\alpha, \beta)=U_{0}\left(v_{1}(\alpha), v_{2}(\beta)\right)$ for all $(\alpha, \beta) \in \mathbb{R}^{2}$ and substituting $V$ into (9), we obtain (7). Clearly, (7) is also satisfied if $V$ is subjected to an increasing transformation.

The final characterization theorem in this section strengthens WCA to SCA. Theorem 4 shows that the second-stage aggregator in this case must be linear, and this is true for any number of attributes (greater than or equal to 2). As in Theorems 2 and 3, the first-stage aggregators are generalized Ginis.

Theorem 4. Suppose that $\mathcal{D} \in\left\{\mathcal{M}, \mathcal{M}_{+}, \mathcal{M}_{+}^{*}\right\}$. The binary relation $\succeq$ on $\mathcal{D}$ satisfies ORD, CONT, MON, ANON, WUPM, SSEP, and SCA if and only if there exists an $n \times q$ matrix $A$ of positive coefficients with $a_{\cdot j}$ nondecreasing and $\sum_{i=1}^{n} a_{i j}=1$ for all $j \in Q$ and a vector $\gamma \in \mathbb{R}_{++}^{q}$ with $\sum_{i=1}^{q} \gamma_{j}=1$ such that

$$
X \succeq Y \Leftrightarrow \sum_{j=1}^{q}\left(\gamma_{j} \sum_{i=1}^{n} a_{i j} \tilde{x}_{i 1}\right) \geq \sum_{j=1}^{q}\left(\gamma_{j} \sum_{i=1}^{n} a_{i j} \tilde{y}_{i 1}\right), \forall X, Y \in \mathcal{D} .
$$

Furthermore, the matrix of coefficients $A$ and the vector $\gamma$ are unique. 
Proof. It is straightforward to show necessity, so we only establish the sufficiency part of the theorem.

We first show that ORD, CONT, and SCA imply that $\succeq$ can be represented by a linear functional on $\mathcal{D}^{\mathrm{D}}$, the set of nonincreasing comonotonic matrices in $\mathcal{D}$. For $X \in \mathcal{D}^{\mathrm{D}}$, let $E(X)=\{Y \in \mathcal{D} \mid Y \sim X\}$ and $B(X)=\{Y \in$ $\mathcal{D} \mid Y \succ X\}$. By applying the argument used by Weymark (1981) in the proof of his Theorem 3, it follows that $E(X)$ is a convex set. ${ }^{16}$ By interpreting elements of $\mathcal{D}^{\mathrm{D}}$ as vectors in $\mathbb{R}^{n q}$, MON then implies that $E(X)$ is the restriction of an $(n q-1)$-dimensional hyperplane in $\mathbb{R}^{n q}$ to $\mathcal{D}^{\mathrm{D}}$. Hence, the indifference contours of $\succeq$ in $\mathcal{D}^{\mathrm{D}}$ are parallel $(q n-1)$-dimensional hyperplanes. For an arbitrary $Z \in B(X)$, we choose a matrix $B \in \mathcal{M}$ in the subspace of normals to $E(X)$ so that $\sum_{i=1}^{n} \sum_{j=1}^{q} b_{i j} z_{i j}>\sum_{i=1}^{n} \sum_{j=1}^{q} b_{i j} x_{i j}$. Thus,

$$
X \succeq Y \Leftrightarrow \sum_{i=1}^{n} \sum_{j=1}^{q} b_{i j} x_{i j} \geq \sum_{i=1}^{n} \sum_{j=1}^{q} b_{i j} y_{i j}, \forall X, Y \in \mathcal{D}^{\mathrm{D}} .
$$

Because SCA implies WCA, Theorem 2 applies if $q>2$ and Theorem 3 applies if $q=2$. Consider any $X, X^{\prime} \in \mathcal{D}$ for which each column of $X^{\prime}$ can be obtained by a permutation of the corresponding column of $X$. It then follows from either (2) or (7) that $X \sim X^{\prime}$. Hence,

$$
X \succeq Y \Leftrightarrow \sum_{i=1}^{n} \sum_{j=1}^{q} b_{i j} \tilde{x}_{i j} \geq \sum_{i=1}^{n} \sum_{j=1}^{q} b_{i j} \tilde{y}_{i j}, \forall X, Y \in \mathcal{D} .
$$

In order for (11) to be consistent with (2) and (7), the functions $v_{j}, j \in N$, in (2) and the function $V$ in (7) must be linear. Thus, (10) holds. The uniqueness of $A$ and $\gamma$ then follow from the corresponding uniqueness results in Theorems 2 and 3.

The key insight underlying the proof of Theorem 4 is that ORD, CONT, and SCA imply that $\succeq$ can be represented by a linear functional on the set of nonincreasing comonotonic matrices in $\mathcal{D}$. This is only consistent with what has been established in Theorems 2 and 3 if the second-stage aggregator function is linear.

\footnotetext{
${ }^{16}$ Weymark's argument is for the set of nonincreasing vectors in $\mathbb{R}_{+}^{n}$, but his argument applies equally well to the domain $\mathcal{D}^{\mathrm{D}}$.
} 


\section{Invariance Axioms}

In the normative approach to inequality measurement for one-dimensional distributions, an inequality index is derived from a social evaluation function. (See Section 8.) A relative inequality index is invariant to a proportional change in all incomes, whereas an absolute inequality index is invariant if a common amount is added to or subtracted from all incomes. In order for an inequality index to be a relative (resp. absolute) index, the underlying social evaluation ordering must be homothetic (resp. translatable). The axioms introduced in this section provide multi-attribute generalizations of these invariance properties for the social evaluation ordering $\succeq$.

Weak Homotheticity simply extends the requirement that the social evaluation ordering be homothetic to the multi-attribute case.

Weak Homotheticity (WHOM). For all $X, Y \in \mathcal{D}$ and all $\lambda>0$,

$$
X \succeq Y \Leftrightarrow \lambda X \succeq \lambda Y
$$

If there is only one attribute, homotheticity of the social evaluation ordering is equivalent to requiring that the ordering be invariant to any change in the units in which the attribute is measured. Tsui (1995) has suggested that the same invariance property should hold in the multi-attribute case. In other words, independent changes in the units in which different attributes are measured should not affect the social evaluation ordering, a property we call Strong Homotheticity.

Strong Homotheticity (SHOM). For all $X, Y \in \mathcal{D}$ and all $q \times q$ diagonal matrices $\Lambda$ for which $\lambda_{j j}>0$ for all $j \in Q$,

$$
X \succeq Y \Leftrightarrow X \Lambda \succeq Y \Lambda \text {. }
$$

$\mathrm{SHOM}$ is a natural assumption if the attributes are different kinds of goods, such as income and life expectancy. However, if different attributes are incomes in different states or time periods, then the attributes should be measured in the same units, in which case SHOM is inappropriate.

The multi-attribute analogue of the requirement that the social evaluation ordering be translatable is Weak Translatability. 
Weak Translatability (WTRA). For all $X, Y \in \mathcal{D}$ and all $\lambda \in \mathbb{R}$ for which $X+\lambda \mathbf{1} \in \mathcal{D}$ and $Y+\lambda \mathbf{1} \in \mathcal{D}$,

$$
X \succeq Y \Leftrightarrow X+\lambda \mathbf{1} \succeq Y+\lambda \mathbf{1}
$$

In the one-attribute case, translatability of the social evaluation ordering is equivalent to requiring the ordering to be invariant to any change in the origin from which the quantity of the attribute is measured. Strong Translatability, an axiom proposed by Tsui (1995), extends this condition by requiring that the social evaluation ordering be invariant to independent changes in the origins from which the quantities of the various attributes are measured. As with SHOM, this condition is inappropriate if there are attributes that should be measured using the same scale.

Strong Translatability (STRA). For all $X, Y \in \mathcal{D}$ and all $q \times q$ diagonal matrices $\Lambda$ for which $X+\mathbf{1} \Lambda \in \mathcal{D}$ and $Y+\mathbf{1} \Lambda \in \mathcal{D}$,

$$
X \succeq Y \Leftrightarrow X+1 \Lambda \succeq Y+1 \Lambda .
$$

These four invariance axioms are closely related to axioms used in the literature on social choice with interpersonal comparisons of utility. ${ }^{17}$ In this literature, a social welfare ordering is defined on distributions of utilities, one for each person. A social welfare ordering is ratio-scale measurable and fully comparable if the ranking of any two utility vectors is invariant to a proportional scaling of all utilities and it is ratio-scale measurable if the factor of proportionality can be person-specific. Similarly, a social welfare ordering is translation-scale measurable and fully comparable if the ranking of any two utility vectors is invariant when a common amount is added to or subtracted from all utilities and it is translation-scale measurable when the amounts added or subtracted can be person-specific. Although social welfare orderings are defined on vectors of utilities and the social evaluation orderings considered here are defined on allocation matrices, we are nevertheless able to exploit social choice theorems that use these ratio-scale and translation-scale axioms in Sections 6 and 7 to help characterize classes of multidimensional generalised Gini relative and absolute inequality indices.

\footnotetext{
${ }^{17}$ For an introduction to this literature, see Bossert and Weymark (1996).
} 


\section{Homothetic Social Evaluation Orderings}

Normative indices of relative inequality are constructed using homothetic social evaluation orderings. In this section, for each of the theorems in Section 4 , we consider the implications of also requiring the social evaluation function to satisfy one of our homotheticity axioms. When defining relative inequality indices, it is customary to assume that there is a positive amount of each attribute. Accordingly, in this section, we suppose that the domain of the social evaluation ordering is $\mathcal{M}_{+}^{*}$.

We begin by assuming that there are at least three attributes and supplement the axioms in Theorem 2 with WHOM. In this case, WHOM places strong restrictions on the functional form of the second-stage aggregator - it must be a mean of order $r$ function. In other words, the function that aggregates the values of the generalized Ginis for the $q$ attributes must be a constant-elasticity-of-substitution function. When $r=0$, this aggregator is a Cobb-Douglas function.

Theorem 5. If $q>2$, then the binary relation $\succeq$ on $\mathcal{M}_{+}^{*}$ satisfies ORD, CONT, MON, ANON, WUPM, SSEP, WCA, and WHOM if and only if there exists an $n \times q$ matrix $A$ of positive coefficients with $a_{\cdot j}$ nondecreasing and $\sum_{i=1}^{n} a_{i j}=1$ for all $j \in Q$, a vector $\gamma \in \mathbb{R}_{++}^{q}$ with $\sum_{j=1}^{q} \gamma_{j}=1$, and a scalar $r$ such that

$$
X \succeq Y \Leftrightarrow\left[\sum_{j=1}^{q} \gamma_{j}\left(\sum_{i=1}^{n} a_{i j} \tilde{x}_{i j}\right)^{r}\right]^{\frac{1}{r}} \geq\left[\sum_{j=1}^{q} \gamma_{j}\left(\sum_{i=1}^{n} a_{i j} \tilde{y}_{i j}\right)^{r}\right]^{\frac{1}{r}}, \forall X, Y \in \mathcal{M}_{+}^{*},
$$

if $r \neq 0$ and

$$
X \succeq Y \Leftrightarrow \prod_{j=1}^{q}\left(\sum_{i=1}^{n} a_{i j} \tilde{x}_{i j}\right)^{\gamma_{j}} \geq \prod_{j=1}^{q}\left(\sum_{i=1}^{n} a_{i j} \tilde{y}_{i j}\right)^{\gamma_{j}}, \forall X, Y \in \mathcal{M}_{+}^{*},
$$

if $r=0$. Furthermore, the matrix of coefficients $A$, the vector $\gamma$, and the scalar $r$ are unique.

Proof. The necessity of the axioms is easy to check, so we only establish the sufficiency part of the theorem.

By Theorem 2, (2) must be satisfied. Let $W: \mathbb{R}_{++}^{q} \rightarrow \mathbb{R}$ be defined by setting

$$
W(g)=\sum_{j=1}^{q} v_{j}\left(g_{j}\right), \forall g \in \mathbb{R}_{++}^{q},
$$


where the functions $v_{j}$ are the functions that appear in (2). Because these functions are continuous and increasing, so is $W$. Because $\succeq$ satisfies WHOM, (2) implies that $W$ is ratio-scale measurable and fully comparable. Hence, by Theorem 2 in Blackorby and Donaldson (1982), $W$ must be a mean of order $r$ function. That is, there exists a vector $\gamma \in \mathbb{R}_{++}^{q}$ with $\sum_{j=1}^{q} \gamma_{j}=1$ and a scalar $r$ such that

$$
W(g)=\left[\sum_{j=1}^{q} \gamma_{j}\left(g_{j}\right)^{r}\right]^{\frac{1}{r}}, \forall g \in \mathbb{R}_{++}^{q},
$$

if $r \neq 0$ and

$$
W(g)=\prod_{j=1}^{q}\left(g_{j}\right)^{\gamma_{j}}, \forall g \in \mathbb{R}_{++}^{q},
$$

if $r=0$. It then follows from (2), (14), (15), and (16) that (12) and (13) hold. In view of the normalization adopted for $\gamma$, the parameters $A, \gamma$, and $r$ are unique.

The sufficiency part of the proof of Theorem 5 exploits the fact that we know from Theorem 2 that the social evaluation ordering can be represented by a two-stage aggregator function and that the first-stage aggregators are generalized Ginis. WHOM implies that the second-stage aggregator function is ratio-scale measurable and fully comparable and this permits us to use a result from the social choice literature due to Blackorby and Donaldson (1982) to characterize the set of admissible second-stage aggregators.

The characterization in Theorem 5 makes essential use of our earlier result that the second-stage aggregator is additively separable. If there are only two attributes, the second-stage aggregator need not be separable. As a consequence, when the axioms in Theorem 3 are supplemented with WHOM, the only additional structure placed on the function $V$ in (7) is that it is homothetic. ${ }^{18}$

Our next theorem demonstrates that the second-stage aggregator in Theorem 5 must be a Cobb-Douglas function if WHOM is strengthened to SHOM. Further, this conclusion holds if there are two or more attributes, not just if there are at least three.

Theorem 6. If $q \geq 2$, then the binary relation $\succeq$ on $\mathcal{M}_{+}^{*}$ satisfies ORD, CONT, MON, ANON, WUPM, SSEP, WCA, and SHOM if and only if there

\footnotetext{
${ }^{18}$ To economize on space, we do not state this result formally.
} 
exists an $n \times q$ matrix $A$ of positive coefficients with $a_{\cdot j}$ nondecreasing and $\sum_{i=1}^{n} a_{i j}=1$ for all $j \in Q$ and a vector $\gamma \in \mathbb{R}_{++}^{q}$ with $\sum_{j=1}^{q} \gamma_{j}=1$ such that (13) holds. Furthermore, the matrix of coefficients $A$ and the vector $\gamma$ are unique.

Proof. We only establish the sufficiency part of the theorem as necessity is straightforward to verify.

It follows from Theorems 2 and 3 that there exists an $n \times q$ matrix $A$ of positive coefficients with $a_{\cdot j}$ nondecreasing and $\sum_{i=1}^{n} a_{i j}=1$ for all $j \in Q$ and there exists a continuous increasing function $V: \mathbb{R}_{++}^{q} \rightarrow \mathbb{R}$ such that

$$
\begin{array}{r}
X \succeq Y \Leftrightarrow V\left(\sum_{i=1}^{n} a_{i 1} \tilde{x}_{i 1}, \ldots, \sum_{i=1}^{n} a_{i q} \tilde{x}_{i q}\right) \geq V\left(\sum_{i=1}^{n} a_{i 1} \tilde{y}_{i 1}, \ldots, \sum_{i=1}^{n} a_{i q} \tilde{y}_{i q}\right), \\
\forall X, Y \in \mathcal{M}_{+}^{*} \cdot{ }^{19}
\end{array}
$$

By SHOM, $V$ is ratio-scale measurable. Hence, by Theorem 4 in Tsui and Weymark (1997), $V$ must be a continuous increasing transform of a CobbDouglas function with positive coefficients. That is, $V$ must be a continuous increasing transform of a function of the form given in (16). The uniqueness of $A$ and $\gamma$ (given the normalization rule for $\gamma$ ) follows from Theorems 2 and 3 .

When there are at least three attributes, Theorem 6 is a corollary to Theorem 5. SHOM implies that the second-stage aggregator function is separable, and this is enough separability for the $q>2$ characterization to also hold when there are only two attributes.

The second-stage aggregator function in (10) is linear. Consequently, the social evaluation orderings identified in Theorem 4 also satisfy WHOM. Note that, when $q>2$, this linear aggregator is obtained by setting $r=1$ in Theorem 5. However, if SHOM is added to the axioms in Theorem 4, an impossibility theorem is obtained because having a linear second-stage aggregator is inconsistent with Theorem 6 .

Theorem 7. If $q \geq 2$, there is no binary relation $\succeq$ on $\mathcal{M}_{+}^{*}$ that satisfies ORD, CONT, MON, ANON, WUPM, SSEP, SCA, and SHOM.

Proof. On the contrary, suppose that there exists a $\succeq$ that satisfies these eight axioms. Then, by Theorem 4, (10) must hold and by Theorem 6, (13) must hold. However, (10) and (13) are inconsistent.

\footnotetext{
${ }^{19}$ When $q>2$, we also know from Theorem 2 that $V$ is additive.
} 


\section{Translatable Social Evaluation Orderings}

Normative indices of absolute inequality are constructed from translatable social evaluation orderings. In this section, we provide the corresponding results for translatable social evaluation orderings to those established in the preceding section for homothetic social evaluation orderings. For simplicity, we now suppose that the domain of the social evaluation ordering is $\mathcal{M}$.

If WTRA is used instead of WHOM in Theorem 5 and the domain is changed from $\mathcal{M}_{+}^{*}$ to $\mathcal{M}$, then the second-stage aggregator must be a KolmPollak function. Of particular note is that linear aggregation functions are members of this class.

Theorem 8. If $q>2$, then the binary relation $\succeq$ on $\mathcal{M}$ satisfies ORD, CONT, MON, ANON, WUPM, SSEP, WCA, and WTRA if and only if there exists an $n \times q$ matrix $A$ of positive coefficients with $a_{\cdot j}$ nondecreasing and $\sum_{i=1}^{n} a_{i j}=1$ for all $j \in Q$, a vector $\gamma \in \mathbb{R}_{++}^{q}$, and a scalar $r$ such that

$$
\begin{array}{r}
X \succeq Y \Leftrightarrow \frac{1}{r} \ln \left[\sum_{j=1}^{q} \gamma_{j} \exp \left(r \sum_{i=1}^{n} a_{i j} \tilde{x}_{i j}\right)\right] \geq \frac{1}{r} \ln \left[\sum_{j=1}^{q} \gamma_{j} \exp \left(r \sum_{i=1}^{n} a_{i j} \tilde{y}_{i j}\right)\right], \\
\forall X, Y \in \mathcal{M},
\end{array}
$$

if $r \neq 0$ and

$$
X \succeq Y \Leftrightarrow \sum_{j=1}^{q} \gamma_{j}\left(\sum_{i=1}^{n} a_{i j} \tilde{x}_{i j}\right) \geq \sum_{j=1}^{q} \gamma_{j}\left(\sum_{i=1}^{n} a_{i j} \tilde{y}_{i j}\right), \forall X, Y \in \mathcal{M},
$$

if $r=0$. Furthermore, the matrix of coefficients $A$, the vector $\gamma$, and the scalar $r$ are unique.

Proof. With the following modifications, the proof of this theorem is the same as the proof of Theorem 5. Because $\succeq$ satisfies WTRA instead of WHOM, the function $W$ (now defined on $\mathbb{R}^{q}$ ) is translation-scale measurable and fully comparable. Hence, by Theorem 3 in Blackorby and Donaldson (1982), W must be a Kolm-Pollak function. That is, there exists a vector $\gamma \in \mathbb{R}_{++}^{q}$ with $\sum_{j=1}^{q} \gamma_{j}=1$ and a scalar $r$ such that

$$
W(g)=\frac{1}{r} \ln \left[\sum_{j=1}^{q} \gamma_{j} \exp \left(r g_{j}\right)\right], \forall g \in \mathbb{R}^{q},
$$


if $r \neq 0$ and

$$
W(g)=\sum_{j=1}^{q} \gamma_{j} g_{j}, \forall g \in \mathbb{R}^{q}
$$

if $r=0$.

If $q=2$, the axioms in Theorem 8 characterize the subset of the social evaluation orderings characterized in Theorem 3 for which the function $V$ in (7) is translatable.

Theorem 9 shows that if STRA is substituted for SHOM in Theorem 6 and the domain is changed from $\mathcal{M}_{+}^{*}$ to $\mathcal{M}$, then the second-stage aggregator must be linear.

Theorem 9. If $q \geq 2$, then the binary relation $\succeq$ on $\mathcal{M}$ satisfies ORD, CONT, MON, ANON, WUPM, SSEP, WCA, and STRA if and only if there exists an $n \times q$ matrix $A$ of positive coefficients with $a_{. j}$ nondecreasing and $\sum_{i=1}^{n} a_{i j}=1$ for all $j \in Q$ and a vector $\gamma \in \mathbb{R}_{++}^{q}$ such that (19) holds. Furthermore, the matrix of coefficients $A$ and the vector $\gamma$ are unique.

Proof. With the following modifications, the proof of this theorem is the same as the proof of Theorem 6. Because $\succeq$ satisfies STRA instead of SHOM, the function $V$ (now defined on $\mathbb{R}^{q}$ ) is translation-scale measurable. Hence, by Theorem 8.1 in Bossert and Weymark (1996), $V$ must be a continuous increasing transform of a function of the form given in (21).

In Theorems 5 and 6 , the second-stage aggregation function represents a binary relation $R$ on $\mathbb{R}_{++}^{q}$. We can define an ordering $R^{*}$ on $\mathbb{R}^{q}$ by setting $u R^{*} v \Leftrightarrow\left(\exp \left(u_{1}\right), \ldots, \exp \left(u_{q}\right)\right) R\left(\exp \left(v_{1}\right), \ldots, \exp \left(v_{q}\right)\right)$ for all $u, v \in \mathbb{R}^{q}$. The ordering $R$ is continuous, increasing, and ratio-scale measurable and fully comparable (resp. ratio-scale measurable) if and only if $R^{*}$ is continuous, increasing, and translation-scale measurable and fully comparable (resp. translation-scale measurable). This observation accounts for why the functional forms of the second-stage aggregators in Theorems 8 and 9 can be obtained from those in Theorems 5 and 6 by a simple exponential change of variables.

Note that the social evaluation orderings identified in Theorem 4 also satisfy STRA (and, hence, WTRA). Thus, Theorem 9 also characterizes all of the social evaluation orderings on $\mathcal{M}$ that satisfy ORD, CONT, MON, ANON, WUPM, SSEP, SCA, and WTRA (resp. STRA). Because the secondstage aggregator is linear, the conflict we found in Theorem 7 with SHOM does not arise if STRA is used instead. 


\section{Multidimensional Inequality Indices}

The Atkinson-Kolm-Sen (see Atkinson (1970), Kolm (1969), and Sen (1973)) and Kolm (1969) procedures for constructing univariate indices of relative and absolute inequality, respectively, both employ a particular representation of the social evaluation function known as the equally-distributed-equivalent income function. The equally-distributed-equivalent income associated with a given univariate income distribution is the per capita income which, if distributed equally, is indifferent to the actual income distribution according to the social evaluation ordering. The equally-distributed-equivalent income function is the mapping that assigns the equally-distributed-equivalent income to each income distribution in thedomain. The Atkinson-Kolm-Sen inequality index measures inequality by computing the ratio of the equallydistributed-equivalent income to the mean income and subtracting this value from 1 . This index is a relative index if the underlying social evaluation function is homothetic. The Kolm inequality index measures inequality by the difference between the mean income and the equally-distributed-equivalent income. This index is an absolute index if the underlying social evaluation function is translatable.

In this section, we describe how the multi-attribute generalizations of the Atkinson-Kolm-Sen and Kolm inequality indices due to Kolm (1977) and Tsui (1995), respectively, are constructed. We also derive the functional forms of these indices for some of the social evaluation orderings characterized in the preceding sections. For further discussion of this approach to the measurement of multidimensional inequality, see Tsui (1995) and Weymark (1999; 2003).

As a domain, we use $\mathcal{M}_{+}^{*}$ when we consider relative inequality indices and we use $\mathcal{M}$ when we consider absolute inequality indices. Throughout this section, we suppose that the social evaluation function $\succeq$ satisfies the following basic properties: ORD, CONT, MON, ANON, and WUPM.

We begin by considering relative indices of inequality. In the univariate case, the value of the Atkinson-Kolm-Sen inequality index for a given distribution has a natural interpetation. It is the fraction of the aggregate income that could be destroyed if incomes are equalized and the resulting distribution is socially indifferent to the original distribution. The Kolm (1977) multi-attribute generalization of this index measures the inequality of an allocation by the fraction of the aggregate amount of each attribute that could be destroyed if every attribute is equalized and the resulting allocation 
is indifferent to the original allocation according to $\succeq$.

Formally, we first define the function $\Delta_{\mathrm{R}}: \mathcal{M}_{+}^{*} \rightarrow \mathbb{R}$ by setting, for each $X \in \mathcal{M}_{+}^{*}, \Delta_{\mathrm{R}}(X)$ equal to the scalar that solves

$$
\Delta_{\mathrm{R}}(X) X_{\mu} \sim X^{20}
$$

Our assumptions on $\succeq$ ensure that $\Delta_{\mathrm{R}}$ is well-defined. The multi-attribute Kolm inequality index associated with $\succeq$ is the function $I_{\mathrm{R}}: \mathcal{M}_{+}^{*} \rightarrow \mathbb{R}$ defined by setting

$$
I_{\mathrm{R}}(X)=1-\Delta_{\mathrm{R}}(X), \forall X \in \mathcal{M}_{+}^{*} .
$$

If $\succeq$ satisfies WHOM, then $I_{\mathrm{R}}$ is a relative index.

It is easy to determine the functional form of the multi-attribute Kolm inequality index that corresponds to each of the social evaluation orderings identified in Section 6. For example, when $\succeq$ is defined as in (12), then (22) is satisfied if

$$
\left[\sum_{j=1}^{q} \gamma_{j}\left(\sum_{i=1}^{n} a_{i j} \Delta_{\mathrm{R}}(X) \mu\left(x_{\cdot j}\right)\right)^{r}\right]^{\frac{1}{r}}=\left[\sum_{j=1}^{q} \gamma_{j}\left(\sum_{i=1}^{n} a_{i j} \tilde{x}_{i j}\right)^{r}\right]^{\frac{1}{r}}, \forall X \in \mathcal{M}_{+}^{*} .
$$

Hence,

$$
\Delta_{\mathrm{R}}(X)=\frac{\left[\sum_{j=1}^{q} \gamma_{j}\left(\sum_{i=1}^{n} a_{i j} \tilde{x}_{i j}\right)^{r}\right]^{\frac{1}{r}}}{\left[\sum_{j=1}^{q} \gamma_{j} \mu\left(x_{\cdot j}\right)^{r}\right]^{\frac{1}{r}}}, \forall X \in \mathcal{M}_{+}^{*}
$$

Therefore,

$$
I_{\mathrm{R}}(X)=1-\frac{\left[\sum_{j=1}^{q} \gamma_{j}\left(\sum_{i=1}^{n} a_{i j} \tilde{x}_{i j}\right)^{r}\right]^{\frac{1}{r}}}{\left[\sum_{j=1}^{q} \gamma_{j} \mu\left(x_{\cdot j}\right)^{r}\right]^{\frac{1}{r}}}, \forall X \in \mathcal{M}_{+}^{*},
$$

is the multi-attribute Kolm inequality index corresponding to (12). This index has a particularly simple form if $r=1, \gamma_{j}=1 / q$ for all $j \in Q$ (so each attribute receives the same weight), and the Gini social evaluation function is used as the first-stage aggregator for each attribute. In this case, (24) becomes

$$
I_{\mathrm{R}}(X)=\frac{\sum_{j=1}^{q} \mu\left(x_{\cdot j}\right) I_{\mathrm{RG}}\left(x_{\cdot j}\right)}{\sum_{j=1}^{q} \mu\left(x_{\cdot j}\right)}, \forall X \in \mathcal{M}_{+}^{*},
$$

\footnotetext{
${ }^{20}$ Recall that $X_{\mu}$ is the allocation matrix for which every entry in the $j^{\text {th }}$ column is equal to $\mu\left(x_{\cdot j}\right)$.
} 
where $I_{\mathrm{RG}}\left(x_{. j}\right)$ is the relative Gini inequality index evaluated at the distribution $x_{. j}$. This index differs from the arithmetic mean of the relative Gini inequality indices for the individual attributes, which Koshevoy and Mosler (1997, p. 275) describe as being a "popular approach" to measuring multidimensional inequality, because the weights in (25) depend on the relative quantities of the attributes.

Similar calculations show that the multi-attribute Kolm inequality index corresponding to (13) is

$$
I_{\mathrm{R}}(X)=1-\frac{\prod_{j=1}^{q}\left(\sum_{i=1}^{n} a_{i j} \tilde{x}_{i j}\right)^{\gamma_{j}}}{\prod_{j=1}^{q} \mu\left(x_{\cdot j}\right)^{\gamma_{j}}}, \forall X \in \mathcal{M}_{+}^{*} .
$$

If $\gamma_{j}=1 / q$ for all $j \in Q$ and the Gini social evaluation function is used as the first-stage aggregator for each attribute, then (26) simplifies to

$$
I_{\mathrm{R}}(X)=1-\prod_{j=1}^{q}\left(E_{\mathrm{RG}}\left(x_{\cdot j}\right)\right)^{\frac{1}{q}}, \forall X \in \mathcal{M}_{+}^{*},
$$

where $E_{\mathrm{RG}}\left(x_{\cdot j}\right)=1-I_{\mathrm{RG}}\left(x_{\cdot j}\right)$ is the relative Gini equality index evaluated at the distribution $x_{\cdot j}$.

We now consider absolute indices of inequality. The univariate Kolm inequality index is equal to the amount of income that could be taken away from every individual in order to obtain a distribution that is socially indifferent to the original distribution if incomes are equalized. The generalization of this index proposed by Tsui (1995) measures inequality by the amount of each attribute that could be taken away from every individual in order to obtain an allocation that is indifferent to the original allocation according to $\succeq$ if the distribution of each attribute is equalized.

To define Tsui's index formally, we first define the function $\Delta_{\mathrm{A}}: \mathcal{M} \rightarrow \mathbb{R}$ by setting, for each $X \in \mathcal{M}, \Delta_{\mathrm{A}}(X)$ equal to the scalar that solves

$$
X_{\mu}-\Delta_{\mathrm{A}}(X) \mathbf{1} \sim X
$$

Our assumptions on $\succeq$ ensure that $\Delta_{\mathrm{A}}$ is well-defined. The multi-attribute Tsui inequality index associated with $\succeq$ is the function $I_{\mathrm{A}}: \mathcal{M} \rightarrow \mathbb{R}$ defined by setting

$$
I_{\mathrm{A}}(X)=\Delta_{\mathrm{A}}(X), \forall X \in \mathcal{M} .
$$

If $\succeq$ satisfies WTRA, then $I_{\mathrm{A}}$ is an absolute index. 
We illustrate the construction of $I_{\mathrm{A}}$ using the social evaluation orderings in (18) and (19). In the case of (18), (28) is satisfied if

$$
\begin{aligned}
\frac{1}{r} \ln \left[\sum_{j=1}^{q} \gamma_{j} \exp \left(r \sum_{i=1}^{n} a_{i j}\left[\mu\left(x_{\cdot j}\right)-\Delta_{\mathrm{A}}(X)\right]\right)\right]= \\
\frac{1}{r} \ln \left[\sum_{j=1}^{q} \gamma_{j} \exp \left(r \sum_{i=1}^{n} a_{i j} \tilde{x}_{i j}\right)\right], \forall X \in \mathcal{M} .
\end{aligned}
$$

Hence, by (29), the multi-attribute Tsui inequality index corresponding to (18) is given by

$$
I_{\mathrm{A}}(X)=\Delta_{\mathrm{A}}(X)=\frac{1}{r} \ln \left[\frac{\sum_{j=1}^{q} \gamma_{j} \exp \left(r \mu\left(x_{\cdot j}\right)\right)}{\sum_{j=1}^{q} \gamma_{j} \exp \left(r \sum_{i=1}^{n} a_{i j} \tilde{x}_{i j}\right)}\right], \forall X \in \mathcal{M} .
$$

Similarly, the multi-attribute Tsui inequality index corresponding to (19) is

$$
I_{\mathrm{A}}(X)=\Delta_{\mathrm{A}}(X)=\sum_{j=1}^{q} \gamma_{j}\left[\mu\left(x_{\cdot j}\right)-\sum_{i=1}^{n} a_{i j} \tilde{x}_{i j}\right], \forall X \in \mathcal{M} .
$$

If $\gamma_{j}=1 / q$ for all $j \in Q$ and the Gini social evaluation function is used as the first-stage aggregator for each attribute, then (31) simplifies to

$$
I_{\mathrm{A}}(X)=\sum_{j=1}^{q}\left[\frac{I_{\mathrm{AG}}\left(x_{\cdot j}\right)}{q}\right], \forall X \in \mathcal{M},
$$

where $I_{\mathrm{AG}}\left(x_{\cdot j}\right)$ is the absolute Gini inequality index evaluated at the distribution $x_{\cdot j}$. Thus, in contrast to the relative case, taking the arithmetic average of the absolute Gini inequality indices for the individual attributes yields a multi-attribute index of absolute inequality that is consistent with our approach.

\section{Correlation Increasing Majorization}

WUPM and WUM are multi-attribute generalizations of the Pigou-Dalton transfer principle. They ensure that the social evaluation is inequality averse in the sense that mean-preserving decreases in the spreads of the attribute distributions are socially desirable. WUPM and WUM only capture one aspect of inequality aversion. When there is more than one attribute, inequality 
can also be decreased by reducing the positive dependence between the rows of the allocation matrix. In this section, we consider one way in which a social evaluation ordering can be required to exhibit this kind of inequality aversion. $^{21}$

The positive dependence between the rows of an allocation matrix can be increased by rearranging two individuals' allocations in such a way that one of these individuals receives at least as much of every attribute as the other. Tsui (1999) calls such a rearrangement a correlation-increasing transfer.

Definition. For all $X, Y \in \mathcal{D}, Y$ is obtained from $X$ by a correlationincreasing transfer if $X \neq Y$ and there exist $i_{1}, i_{2} \in N$ such that (i) $y_{i_{1} j}=$ $\min \left\{x_{i_{1} j}, x_{i_{2} j}\right\}$ for all $j \in Q$, (ii) $y_{i_{2} j}=\max \left\{x_{i_{1} j}, x_{i_{2} j}\right\}$ for all $j \in Q$, and (iii) $y_{i}$. $=x_{i}$. for all $i \notin\left\{i_{1}, i_{2}\right\}$.

By considering finite sequences of such transfers, we can define the following partial order on the set of allocation matrices $\mathcal{D}$.

Definition. For all $X, Y \in \mathcal{D}, Y$ is more correlated than $X$, denoted $Y \succ_{\mathrm{C}}$ $X$, whenever $Y$ can be obtained from $X$ by a finite sequence of correlationincreasing transfers.

A sequence of correlation-increasing transfers increases inequality and preserves the mean value of each attribute. Hence, it is socially undesirable.

Correlation Increasing Majorization (CIM). For all $X, Y \in \mathcal{D}$,

$$
Y \succ_{\mathrm{C}} X \Rightarrow X \succ Y \text {. }
$$

CIM was introduced into the inequality literature by Tsui (1999). It is based on the concept of a multivariate arrangement increasing function due to Boland and Proschan (1988). Reservations about this axiom have been raised by Bourguignon and Chakravarty (2003) because CIM does not take account of individual preferences. They note that if everyone has the same utility function and there are two attributes, then the value of a utilitarian social evaluation function decreases if the attributes are complements (it increases if the attributes are substitutes), which is inconsistent with CIM. See Tsui (1999) for further discussion of this axiom and its relationship to similar principles used in statistics and in the measurement of risk.

\footnotetext{
${ }^{21}$ For an overview of different positive dependency concepts, see Joe (1997).
} 
Note that CIM is stated in terms of the strict social evaluation relation $\succ$, whereas WUPM and WUM use the weak relation $\succeq$. Strong versions of the latter axioms can be obtained by replacing $\succeq$ with $\succ$ in their definitions.

CIM is a majorization axiom that has no univariate counterpart. By its very definition, CIM takes account of the dependencies that exist between the distributions of different attributes. The theorems in the preceding sections all employ SSEP as one of their axioms. SSEP requires the conditional distribution of any attribute to be independent of the distributions of the other attributes. This separability of the social evaluation ordering $\succeq$ across attributes creates a tension between CIM and SSEP. This tension is also present if SSEP is weakened to WSEP; i.e., if only one attribute is required to be separable from the other attributes. Theorem 10 shows that this conflict is fundamental: If $\succeq$ satisfies ANON, it is not possible to satisfy both CIM and WSEP.

Theorem 10. Suppose that $\mathcal{D} \in\left\{\mathcal{M}, \mathcal{M}_{+}, \mathcal{M}_{+}^{*}\right\}$. Then, there does not exist a binary relation $\succeq$ on $\mathcal{D}$ that satisfies ANON, WSEP, and CIM.

Proof. Let $\succeq$ be a binary relation on $\mathcal{D}$ satisfying WSEP and ANON. By WSEP, there exists an attribute $j_{0}$ such that $j_{0}$ is separable from the attributes in $Q \backslash\left\{j_{0}\right\}$. Let $X \in \mathcal{D}$ be defined by setting (i) $x_{i j}=1$ for all $j \neq j_{0}$ and all $i \in N$ and (ii) $x_{i j_{0}}=i$ for all $i$. Define $Y \in \mathcal{D}$ by setting (i) $y_{i j}=x_{i j}$ for all $j \neq j_{0}$ and all $i \in N$ and (ii) $y_{i j_{0}}=n-i+1$ for all $i \in N$. Note that $Y$ is obtained from $X$ by a permutation of the rows of $X$. Hence, by ANON, $X \sim Y$. Now, consider the matrices $X^{\prime}$ and $Y^{\prime}$ obtained from $X$ and $Y$, respectively, by replacing, for all $j \neq j_{0}$ and all $i \in N, x_{i j}$ and $y_{i j_{0}}$ with $i$. Because $j_{0}$ is separable from $Q \backslash\left\{j_{0}\right\}, X \sim Y$ implies that $X^{\prime} \sim Y^{\prime}$. The columns of $X^{\prime}$ are all identical, whereas those of $Y^{\prime}$ are not. Consequently, $X^{\prime} \succ_{\mathrm{C}} Y^{\prime}$, which violates CIM.

The argument used in the proof of Theorem 10 can be illustrated quite simply when $n=q=3$. For concreteness, suppose that attribute 3 is separable from attributes 1 and 2. By ANON, we have

$$
X=\left(\begin{array}{lll}
1 & 1 & 1 \\
1 & 1 & 2 \\
1 & 1 & 3
\end{array}\right) \sim\left(\begin{array}{lll}
1 & 1 & 3 \\
1 & 1 & 2 \\
1 & 1 & 1
\end{array}\right)=Y
$$

By WSEP, indifference is preserved if the first two columns of $X$ and $Y$ are replaced by any other entries, provided that the replacement entries are the 
same, component by component, in both matrices. In particular, this is the case if each of these columns is replaced with the third column of $X$. Thus,

$$
X^{\prime}=\left(\begin{array}{lll}
1 & 1 & 1 \\
2 & 2 & 2 \\
3 & 3 & 3
\end{array}\right) \sim\left(\begin{array}{lll}
1 & 1 & 3 \\
2 & 2 & 2 \\
3 & 3 & 1
\end{array}\right)=Y^{\prime}
$$

The columns of $X^{\prime}$ are perfectly correlated, whereas the last column of $Y^{\prime}$ differs from the first two. As a consequence, $X^{\prime}$ is more correlated than $Y^{\prime}$. To satisfy CIM, we would have to have $Y^{\prime} \succ X^{\prime}$, which is not the case.

As noted in Section 2, the framework used here has also been employed to analyze the measurement of inequality under uncertainty. In this interpretation, the $i j^{\text {th }}$ entry in an allocation matrix is the income (or the interpersonally comparable utility) of individual $i$ in state $j$. An important feature of this model is that the units in which incomes in different states are measured are the same. If $\succeq$ is interpreted as being the preference relation of a social decision-maker, then Theorem 10 implies that CIM must be violated if $\succeq$ treats individuals symmetrically and it respects the axioms of expected utility theory applied to allocation matrices. This follows because the expected utility axioms include an independence assumption that implies SSEP and, hence, WSEP. This raises the question as to which, if any, model of decision-making under uncertainty is compatible with ANON and CIM.

Schmeidler (1989) has suggested representing a preference over uncertain outcomes by a Choquet integral with respect to a non-additive measure. This model does not employ the independence assumption of expected utility theory. Nevertheless, by adapting an example due to Ben-Porath, Gilboa, and Schmeidler (1997), we are able to show that Schmeidler's proposal is inconsistent with CIM if $\succeq$ satisfies ANON.

Suppose that $\succeq$ is represented by a Choquet functional $V$ on $\mathcal{D}$. A subset $\mathcal{S}$ of $\mathcal{D}$ is comonotonic if for all $X, Y \in \mathcal{S}, x_{i j}>x_{i^{\prime} j^{\prime}}$ implies that $y_{i j} \geq y_{i^{\prime} j^{\prime}}{ }^{22}$ The most relevant feature here of a Choquet functional is that it is linear on any cone of comonotonic allocation matrices. Suppose that $n=q=2$. By ANON, we have

$$
X=\left(\begin{array}{ll}
1 & 0 \\
0 & 0
\end{array}\right) \sim\left(\begin{array}{ll}
0 & 0 \\
1 & 0
\end{array}\right)=Y .
$$

\footnotetext{
${ }^{22}$ Note that this definition implicitly assumes that the $n q$ variables are all measured in the same units.
} 
If $Z$ is comonotonic with both $X$ and $Y$, then the linearity of $V$ implies that $V(X+Z)=V(X)+V(Z)$ and $V(Y+Z)=V(Y)+V(Z)$, from which it follows that $V(X+Z)=V(Y+Z)$. The matrix

$$
Z=\left(\begin{array}{ll}
1 & 1 \\
1 & 0
\end{array}\right)
$$

is such a matrix. Hence, $X \sim Y$ implies that

$$
X^{\prime}=\left(\begin{array}{ll}
2 & 1 \\
1 & 0
\end{array}\right) \sim\left(\begin{array}{ll}
1 & 1 \\
2 & 0
\end{array}\right)=Y^{\prime},
$$

where $X^{\prime}=X+Z$ and $Y^{\prime}=Y+Z$. Clearly, $X^{\prime} \succ_{\mathrm{C}} Y^{\prime}$. Therefore, provided that individuals are treated symmetrically, Schmeidler's model is not compatible with CIM. ${ }^{23}$

Ben-Porath, Gilboa, and Schmeidler (1997) have suggested that instead of using Schmeidler's model when measuring inequality under uncertainty, one should instead represent $\succeq$ by a multiple-priors (min-of-means) functional. A functional $V$ on $\mathcal{D}$ is a multiple-priors functional if there exists a compact and convex set $\mathcal{C}$ of probability measures over the product space $N \times Q$ such that for all $X \in \mathcal{D}, V(X)=\min _{p \in \mathcal{C}} \sum_{i, j} p_{i j} x_{i j}$, where $p_{i j}$ is the probability put by $p$ on the $i j^{\text {th }}$ entry in an allocation matrix. This kind of functional was introduced by Gilboa and Schmeidler (1989). The multiple-priors model is flexible enough to be compatible with both ANON and CIM.

More precisely, Ben-Porath, Gilboa, and Schmeidler (1997) have suggested the following procedure. Let $J_{1}$ be a multiple-priors functional defined on $\mathbb{R}^{n}$, and $J_{2}$ be a multiple-priors functional defined on $\mathbb{R}^{q}$. They then define $\left(J_{1} * J_{2}\right)$ as follows: for all $X \in \mathcal{D},\left(J_{1} * J_{2}\right)(X)$ is obtained by first applying $J_{2}$ over each row of $X$, and then applying $J_{1}$ to the $n$-dimensional vector obtained by this mean. The functional $J_{2} * J_{1}$ is defined similarly, by permuting the role of $J_{1}$ and $J_{2}$. Ben-Porath, Gilboa, and Schmeidler then suggest to consider as a social evaluation function the functional

\footnotetext{
${ }^{23}$ Koshevoy and Mosler (1997) have proposed two multi-attribute extensions of the Gini index of relative inequality. One of these indices is based on the characterization of the Gini in terms of the expected relative mean difference of the incomes and the other is based on the characterization of the Gini in terms of the area between the Lorenz curve and the diagonal of a unit square. For each of their indices, it is readily verified that the allocation matrices $X^{\prime}$ and $Y^{\prime}$ are regarded as exhibiting the same degree of inequality even though $X^{\prime}$ is more correlated than $Y^{\prime}$.
} 
$J=\alpha\left(J_{1} * J_{2}\right)+(1-\alpha)\left(J_{2} * J_{1}\right)$, where $\alpha \in[0,1]$ is a fixed parameter. They furthermore prove that $J$ is a multiple-priors functional defined on $\mathcal{D}$. Now, consider the special case where $J_{1}$ is the Gini operator and $J_{2}$ is the expectation operator with respect the the uniform probability distribution over $Q$. Then, $J$ is defined as:

$$
J(X)=\alpha G\left(\frac{1}{q} \sum_{j=1}^{q} x_{1 j}, \cdots, \frac{1}{q} \sum_{j=1}^{q} x_{n j}\right)+(1-\alpha) \sum_{j=1}^{q} \frac{1}{q} G\left(x_{\cdot j}\right)
$$

Observe that the second term of the right-hand side of equation (33) is invariant to any correlation-increasing transfer. On the other hand, if $Y$ is obtained from $X$ by a correlation increasing transfer between individuals $i_{1}$ and $i_{2}$, then the vector $\left(\frac{1}{q} \sum_{j} x_{1 j}, \cdots, \frac{1}{q} \sum_{j} x_{n j}\right)$ strictly dominates the vector $\left(\frac{1}{q} \sum_{j} y_{1 j}, \cdots, \frac{1}{q} \sum_{j} y_{n j}\right)$ for the Lorenz ordering. Hence, if $Y$ is obtained from $X$ by a finite sequence of correlation increasing transfers, $\left(\frac{1}{q} \sum_{j} x_{1 j}, \cdots, \frac{1}{q} \sum_{j} x_{n j}\right)$ strictly dominates the vector $\left(\frac{1}{q} \sum_{j} y_{1 j}, \cdots, \frac{1}{q} \sum_{j} y_{n j}\right)$ for the Lorenz ordering. Since the Gini index is coherent with the Lorenz ordering, we obtain that, in this case,

$$
G\left(\frac{1}{q} \sum_{j} x_{1 j}, \cdots, \frac{1}{q} \sum_{j} x_{n j}\right)>G\left(\frac{1}{q} \sum_{j} y_{1 j}, \cdots, \frac{1}{q} \sum_{j} y_{n j}\right),
$$

which shows that $J$ defined as above is compatible with CIM. It is obvious that $J$ also satisfies ANON.

As a consequence, the more general model considered by Gajdos and Maurin (2002), which essentially allows for $J_{1}$ and $J_{2}$ to be more general functional than multiple-priors, and considers aggregators of $J_{1} * J_{2}$ and $J_{2} * J_{1}$ other than only the convex combination, is also compatible with these CIM and ANON.

\section{Concluding Remarks}

The social evaluation orderings axiomatized in this article all have representations that can be expressed in terms of a two-stage aggregation procedure. In the first stage, the distributions of each attribute are aggregated using generalized Gini social evaluation functions. The value of the representation function is then determined by aggregating these generalized Ginis. When an inequality index is derived from a social evaluation ordering having this 
structure, it is a simple matter to determine the contribution of each attribute to overall inequality. However, as Theorem 10 demonstrates, the cost of having this aggregation property is that it is not possible to satisfy CIM. How serious this drawback is depends on the appeal of CIM, which, as we have noted, is an axiom that has been criticized by Bourguignon and Chakravarty (2003). For those who find CIM appealing, our results provide a benchmark from which to judge the role that axioms like CIM play in determining the functional structure of an inequality index.

The work of List (1999) and Tsui (1999) sheds some light on the classes of inequality indices that satisfy the inequality counterparts of CIM and either WUPM or WUM. However, as List notes, Tsui employs a controversial decomposability axiom. On the other hand, List's indices are all constructed by using a utility function to reduce the problem to one of univariate inequality measurement and this, too, may be seen as being unduly restrictive. There is therefore much scope for further axiomatic investigations of multidimensional inequality.

\section{References}

Atkinson, A. B., 1970. On the measurement of inequality. Journal of Economic Theory 2, 244-263.

Atkinson, A. B., Bourguignon, F., 1982. The comparison of multidimensioned distributions of economic status. Review of Economic Studies 49, 183-201.

Ben-Porath, E., Gilboa, I., Schmeidler, D., 1997. On the measurement of inequality under uncertainty. Journal of Economic Theory 75, 194-204.

Blackorby, C., Bossert, W., Donaldson, D., 1999. Income inequality measurement: The normative approach. In: Silber, J. (Ed.), Handbook of Income Inequality Measurement. Kluwer Academic Publishers, Boston, pp. 133157.

Blackorby, C., Donaldson, D., 1982. Ratio-scale and translation-scale full interpersonal comparability without domain restrictions: Admissible socialevaluation functions. International Economic Review 23, 249-268.

Blackorby, C., Donaldson, D., Auersperg, M., 1981. A new procedure for the measurement of inequality within and among population subgroups. Canadian Journal of Economics 14, 665-685.

Blackorby, C., Primont, D., Russell, R. R., 1978. Duality, Separability, and 
Functional Structure: Theory and Economic Applications. North-Holland, New York.

Boland, P. J., Proschan, F., 1988. Multivariate arrangement increasing functions with applications in probability and statistics. Journal of Multivariate Analysis 25, 286-298.

Bossert, W., Weymark, J. A., 1996. Utility in social choice. In: Barberà, S., Hammond, P. J., Seidl, C. (Eds.), Handbook of Utility Theory, Volume 2: Extensions. Kluwer Academic Publishers, Boston, forthcoming.

Bourguignon, F., Chakravarty, S. R., 2003. The measurement of multidimensional poverty. Journal of Economic Inequality 1, 25-49.

Dardanoni, V., 1995. On multidimensional inequality measurement. In: Dagum, C., Lemmi, A. (Eds.), Income Distribution, Social Welfare, Inequality, and Poverty. Vol. 6 of Research on Economic Inequality. JAI Press, Stamford, CT, pp. 201-207.

Debreu, G., 1954. Representation of a preference ordering by a numerical function. In: Thrall, R. M., Coombs, C. H., Davis, R. L. (Eds.), Decision Processes. John Wiley and Sons, New York, pp. 159-165.

Debreu, G., 1960. Topological methods in cardinal utility theory. In: Arrow, K. J., Karlin, S., Suppes, P. (Eds.), Mathematical Methods in the Social Sciences, 1959. Stanford University Press, Stanford, pp. 16-26.

Dutta, B., 2002. Inequality, poverty, and welfare. In: Arrow, K. J., Sen, A. K., Suzumura, K. (Eds.), Handbook of Social Choice and Welfare. Vol. 1. North-Holland, Amsterdam, pp. 597-633.

Gajdos, T., Maurin, E., 2002. Unequal uncertainties and uncertain inequalities: An axiomatic approach. Journal of Economic Theory, forthcoming.

Gilboa, I., Schmeidler, D., 1989. Maximin expected utility with non-unique prior. Journal of Mathematical Economics 18, 141-153.

Gorman, W. M., 1968. The structure of utility functions. Review of Economic Studies 35, 369-390.

Hardy, G. H., Littlewood, J. E., Pólya, G., 1934. Inequalities. Cambridge University Press, Cambridge.

Joe, H., 1997. Multivariate Models and Dependence Concepts. Chapman and Hall, London.

Kolm, S.-C., 1969. The optimal production of social justice. In: Margolis, J., Guitton, H. (Eds.), Public Economics. Macmillan, London, pp. 145-200.

Kolm, S.-C., 1977. Multidimensional egalitarianisms. Quarterly Journal of Economics 91, 1-13.

Koshevoy, G. A., Mosler, K., 1997. Multivariate Gini indices. Journal of 
Multivariate Analysis 60, 252-276.

List, C., 1999. Multidimensional inequality measurement: A proposal. Working Paper in Economics No. 1999-W27, Nuffield College, Oxford.

Maasoumi, E., 1986. The measurement and decomposition of multidimensional inequality. Econometrica 54, 991-997.

Maasoumi, E., 1999. Multidimensioned approaches to welfare analysis. In: Silber, J. (Ed.), Handbook of Income Inequality Measurement. Kluwer Academic Publishers, Boston, pp. 437-477.

Marshall, A. W., Olkin, I., 1979. Inequalities: Theories of Majorization and Its Applications. Academic Press, New York.

Pollak, R. A., 1971. Additive utility functions and linear Engel curves. Review of Economic Studies 38, 401-414.

Savaglio, E., 2002. Multidimensional inequality: A survey. Working Paper No. 362, Dipartimento di Economia Politica, Università degli Studi di Siena.

Schmeidler, D., 1989. Subjective probability and expected utility without additivity. Econometrica 57, 571-587.

Sen, A. K., 1973. On Economic Inequality. Clarendon Press, Oxford.

Tsui, K.-Y., 1995. Multidimensional generalizations of the relative and absolute inequality indices: The Atkinson-Kolm-Sen approach. Journal of Economic Theory 67, 251-265.

Tsui, K.-Y., 1999. Multidimensional inequality and multidimensional generalized entropy measures: An axiomatic derivation. Social Choice and Welfare 16, 145-157.

Tsui, K.-Y., Weymark, J. A., 1997. Social welfare orderings for ratio-scale measurable utilities. Economic Theory 10, 241-256.

Weymark, J. A., 1981. Generalized Gini inequality indices. Mathematical Social Sciences 1, 409-430.

Weymark, J. A., 1999. Comment on Blackorby, Bossert, and Donaldson (1999). In: Silber, J. (Ed.), Handbook of Income Inequality Measurement. Kluwer Academic Publishers, Boston, pp. 157-161.

Weymark, J. A., 2003. The normative approach to the measurement of multidimensional inequality. Working Paper No. 03-W14, Department of Economics, Vanderbilt University. 